\title{
Bandwidth and Resource Allocation Optimization Through a Probabilistic Algorithm for Mobile TV
}

\author{
Samira Sadeghi* \\ University of Alberta and INVIDI Technologies \\ Corporation \\ Edmonton, AB, Canada \\ ssadeghi@ualberta.ca
}

\author{
Ivan Mizera \\ University of Alberta \\ Edmonton, AB, Canada \\ imizera@ualberta.ca
}

\begin{abstract}
In this paper we consider the problem of bandwidth and resource allocation optimization for delivering addressable advertising in traditional TV. Transmitting addressable advertising over a mobile TV platform via LTE broadcast with eMBMS, which is capable of efficiently supporting a large number of concurrent users within available network and spectrum constraints, has relevant structural similarities to delivering this advertising over traditional television systems, and so these results from the one area transfer to the other. We introduce a probabilistic algorithm for resource optimization and detail its exact recursive $O\left(n^{2}\right)$ (in the number of delivered programming networks) implementation together with a practical approximation. The proposed methods are evaluated on their performance against real historical data on TV programming and viewing, with the new methods showing significant improvement in terms of advertising revenue over methods currently used in the industry.
\end{abstract}

\section{CCS CONCEPTS}

- Mathematics of computing $\rightarrow$ Probabilistic algorithms; Stochastic processes; Nonparametric statistics; • Computing methodologies $\rightarrow$ Gaussian processes;

\section{KEYWORDS}

Optimization, Addressable Advertising, Probability Forecasting and Resource Allocation

ACM Reference Format:

Samira Sadeghi and Ivan Mizera. 2017. Bandwidth and Resource Allocation Optimization Through a Probabilistic Algorithm for Mobile TV. In MoMM '17: The 15th International Conference on Advances in Mobile Computing Multimedia, December 4-6, 2017, Salzburg, Austria. ACM, New York, NY, USA, 7 pages. https://doi.org/10.1145/3151848.3151865

\section{INTRODUCTION}

We propose a probabilistic algorithm for bandwidth and resource allocation in TV media advertising and develop its $O\left(n^{2}\right)$ implementation. Both an approximate and exact recursive solutions are

\footnotetext{
${ }^{*}$ Corresponding Author

Permission to make digital or hard copies of part or all of this work for personal or classroom use is granted without fee provided that copies are not made or distributed for profit or commercial advantage and that copies bear this notice and the full citation on the first page. Copyrights for third-party components of this work must be honored

For all other uses, contact the owner/author(s).

MoMM '17, December 4-6, 2017, Salzburg, Austria

(c) 2017 Copyright held by the owner/author(s).

ACM ISBN 978-1-4503-5300-7/17/12.

https://doi.org/10.1145/3151848.3151865
}

developed. We evaluate the performance of the proposed methods on real historical data from the traditional TV industry. All methods show significant improvement in terms of advertising profit over the existing industrial practice. We explain how our results, which were originally formulated for application to traditional TV distribution, can transfer and provide utility to the rapidly growing scenario of mobile transmission.

Viewing of mobile TV on streaming digital devices is increasing rapidly across the world [4], following improving mobile internet capabilities. Through efficient mobile systems such as LTE and protocols such as Evolved Multimedia Broadcast Multicast Service (eMBMS), mobile TV may be delivered to a great number of users at once within the constraints of network architectures and spectrum. At the same time, advertising has increasingly shifted to efficient addressable advertising models, which provide industry with the opportunity to maximize advertising revenue and profitability and enhance availability of advanced technologies. Continued success of the new TV delivery models will require adaptation of the new addressable advertising models to the mobile technologies while respecting their resource and bandwidth constraints.

As viewing shifts from traditional TV to Mobile TV, advertisers are following. Traditionally delivered television still captures the majority of video-based ad dollars, but its share is shiffting to new digital video models. Transmitting addressable advertising over mobile TV platforms via LTE has relevant structural similarities to over-the-air or cable broadcast, and so there is a real opportunity of adopting addressability to the expanding mobile platform. By considering multicast to be similar to broadcasting, and taking into account the bandwidth constraints of carriers as akin to available channel constraints of traditional television distribution, we can transfer results from the field of fixed channel delivery to solving the problems of mobile TV. Herein, we use the language of traditional TV delivery, but under general assumptions of resource constraint the results apply as well to mobile TV ([1],[2]).

\subsection{Overview of the Addressable Advertising Model}

We take as given that there are some number of available transmission channels which we divide into channels for delivery of programming and a smaller secondary pool dedicated to ad delivery. The limited ad delivery channels are not assigned to particular programming channels, but are assigned as needed in real time. Thus, if many programming networks happen to enter an ad break at the same time, they will conflict in their requests for these limited ad delivery channels. The timing of the breaks is not known until 
shortly before the break airs, so it is not in general possible to predict beforehand what exact collisions between requests will occur [3]. At the same time, once ad delivery channels are allocated to a break, it is not possible to reallocate them to a break on a different network until the first break completes, which takes some finite amount of time during which requests from other networks may arrive. Crucially, the value of handling one break over another may vary widely, due to audience sizes, demographic composition, and current advertiser requirements.

The rest of this paper is organized as follows. In section 2 we describe the problem of interest, present the naive solution as a kind of Greedy algorithm, and introduce a novel Smart Predictive algorithm designed for improved outcomes. The theoretical results are discussed in section 3. Results from simulation experiments using some real-world data appear in section 4. A summary is presented in section 5. References in the current literature ([1],[2],[6]) do not apply directly to the exact circumstance described so we understand our methods to be novel in the application area.

\section{PROBLEM DESCRIPTION AND SOLUTIONS}

INVIDI Technologies Corporation is transforming TV advertising from an undifferentiated delivery to a personalized, accountable experience. The new technologies create systems that multiplex different ads to different viewers who are watching the same programming network, by instructing the individual cable signal converter set-top boxes within each household to transparently select out of the available advertising those ads specifically addressed to the demographics of that household.

This strategy permits an aggregation model of advertising, improving on the linear model. In aggregation, advertisers pick the type of people who they want to see the ad, rather than coordinating with the networks and distributors to pick spots in which the ad will play. The use of aggregation improves the advertising efficiency of spots, measured by the number of ad impressions that are successfully delivered to the correct demographic (rewards), because ads in the linear stream that would otherwise fail to satisfy addressability requirements in some households may be replaced with alternatives that are correctly addressed. Advertisers ultimately pay per impression, so this technique provides the industry with the opportunity to increase advertising revenue/profitability and in return allows for more broad accessibility of programming and advanced technologies.

However, this system imposes a new cost on the cable companies: the loss of some transmission bandwidth to the ad delivery channels. In addition, the number of networks for which the INVIDI system is enabled may be large and due to capacity constraints, there will be only a limited number of shared resources $k$ (often as few as four sets of three or four ad delivery channels), to support incoming breaks. Eventually, some request for resources must be declined. We want to optimize the expected payoff, calculated as the maximum expected number of viewers in serviced network ad breaks over some period of time.

\subsection{Greedy Algorithm vs Smart Predictive Algorithm}

The existing method of resource allocation is a straightforward first-come-first-serve (Greedy) algorithm, which serves networks unconditionally as they place requests until lack of resources forces denial. Obviously, such an algorithm is in general suboptimal: if the serving rewards differ and a highly lucrative network has a big probability of placing a commercial break in the near future, it may be more profitable to reserve the resource for it rather than serving the less rewarding ones. This Greedy algorithm is a base case, and it can be implemented and tested against recorded or synthesized break arrival data, in either a continuous-time or discrete-time version. The question becomes whether additional algorithms can be developed that are expected to perform better than the Greedy algorithm over the long term in real software deployments.

We propose an improved algorithm, Smart Predictive. The concept of the Smart Predictive algorithm is that it proceeds by determining networks that have the highest expected profit values and then computes whether reserving the resources for these networks would have a higher expected value outcome than assigning through Greedy.

The constructed algorithm can be shown theoretically to yield better results when the probabilities of break arrivals are known. If the probabilities are estimated online by the system, experiments on real data show that the Smart Predictive algorithm outperforms the Greedy algorithm at maximizing the payoff defined above.

\section{THEORETICAL RESULT}

We start with a mathematical model elucidated after discussion with experts in the industry. The following assumptions are adopted in this mathematical model:

- Time discretized to one minute.

- Known value of each network per minute (rewards).

- Probabilistic independence of break arrivals between networks.

- Time dependence of probability of break arrivals within a network.

Time discretization by each minute results in localization of decisions and interactions to the individual minute. This model is fully defined by provision of the values for $P_{i}$ and $R_{i} . P_{i}$ is the probability of network $i$ going to break, and the $R_{i}$ is the value of servicing network $i$ if it goes to break, which is simplified to the number of viewers of that network for these experiments. For the discretized case, these values are given for each minute.

Moreover, in order to form an initial solution, we assume that we are down to the last available set of resources $(k=1)$. Hence, more than one network going into break in an overlapping minute will cause conflicts in their requests for the last resource. In our mathematical model we assume that Greedy works as if this resource is allocated randomly (probability of one break coming before the other one is the same as coming after). That is, if out of the $n$ networks, the networks $\left\{n_{1}, n_{2}, \cdots, n_{q}\right\}, q \leq n$, go to break in an overlapping minute, then the expected profit will be $\frac{R_{1}+R_{2}+\cdots+R_{q}}{q}$, which is simply the mean rewards for networks in collision, since we have just one resource to support coming breaks. The Smart 
Predictive algorithm is formulated for the case of known probabilities of break arrival per unit of time per network. This algorithm is derived from fundamental rules of probability calculus [8]. It is not certain that using the new algorithm will always bring an improvement in every decision; however, by the law of large numbers, it will have almost surely superior outcomes on average [5],[8].

\subsection{Basic Notation}

Before moving to the main result, we define our notation that will be used throughout. All variables are time dependent, but to ease the

\begin{tabular}{|c|c|}
\hline$k$ & number of available resources \\
\hline$n$ & number of programming networks \\
\hline $\mathbf{P}_{t}=\left(P_{1 t}, P_{2 t}, P_{3 t}, \ldots\right)$ & $\begin{array}{l}\text { probabilities of networks } 1,2,3, \ldots \text { airing } \\
\text { commercial breaks at time } t\end{array}$ \\
\hline $\mathbf{R}_{t}=\left(R_{1 t}, R_{2 t}, R_{3 t}, \ldots\right)$ & $\begin{array}{l}\text { number of delivered impressions (rewards) } \\
\text { for networks } 1,2,3, \ldots \text { airing commercial } \\
\text { breaks at time } t\end{array}$ \\
\hline$G_{k t}(S, \mathbf{P}, \mathbf{R})$ & $\begin{array}{l}\text { the expected reward of the Greedy algorithm } \\
\text { serving } k \text { resources at time } t \text { for any subset, } \\
S \text {, of networks }\end{array}$ \\
\hline$S P_{k t}(S, \mathbf{P}, \mathbf{R})$ & $\begin{array}{l}\text { the expected reward of the Smart Predictive } \\
\text { algorithm serving } k \text { resources at time } t \text { for } \\
\text { any subset, } S \text {, of networks }\end{array}$ \\
\hline$[i \in A]$ & 1 if $i \in A$ and 0 otherwise \\
\hline$|A|$ & cardinality of set $A$ \\
\hline$S_{\ell}$ & $\begin{array}{l}\text { the set of all subsets of } S \text { with cardinality } \ell \text {; } \\
S_{|S|}=S\end{array}$ \\
\hline \multirow{2}{*}{$\prod_{l=p}^{q} A_{l}$} & $A_{q} A_{q-1} \ldots A_{p}$ if $q \geq p$ or 1 if $p>q$ \\
\hline & $\forall A_{l} \in R^{1}$ \\
\hline
\end{tabular}

notation the time subscript $(t)$ will be dropped. Hence, definitions are per minute. In addition, for any subset, $S$, of networks, we define $G_{k}(S)$ and $S P_{k}(S)$ to be the expected reward of the Greedy algorithm and Smart Predictive algorithm serving $k$ resources at time $t$ - the latter suppressed in the notation, as well as $\mathbf{P}$ and $\mathbf{R}$.

\subsection{Greedy Algorithm Formulation for One Resource}

The expected value of the Greedy algorithm across the span of all networks has a well-defined mathematical formula.

For any subset, $S$, of networks, we define $G_{1}(S)$ to be the expected reward of the Greedy algorithm serving 1 resource at time $t$ (again, the latter suppressed in the notation, as well as $\mathbf{P}$ and $\mathbf{R}$ )

$$
G_{1}(S)=\sum_{\ell=1}^{|S|} \sum_{I \in S_{\ell}} L_{S}(I) \frac{1}{\ell} \sum_{j \in I} R_{j},
$$

where $L_{S}(I)=\prod_{i \in S} P_{i}^{[i \in I]}\left(1-P_{i}\right)^{1-[i \in I]}=\prod_{i \in S \cap I} P_{i} \prod_{j \in S \backslash I}\left(1-P_{j}\right)$.

Note that the term $\ell$ reflects the number of networks going into break in an overlapping minute and $\frac{1}{\ell}$ accounts for the random allocation of the resource.
Furthermore, we can rewrite $G_{1}(S)$ just in terms of $P_{i} \mathrm{~s}$ and $R_{i} \mathrm{~s}$. Assuming, $S=\{1,2, \cdots, n\}$ and $\max _{i=1,2, \cdots, n} P_{i} R_{i}=P_{1} R_{1}$ :

$$
G_{1}(S)=P_{1} R_{1} \Delta+I-P_{1} I I
$$

where

$$
\begin{aligned}
& \Delta=1+\sum_{m=1}^{n-1} \frac{(-1)^{m}}{m+1} \sum_{i_{1}<i_{2}<\cdots<i_{m}} \prod_{j=i_{1}}^{i_{m}} P_{j}, \\
& I=\sum_{m=1}^{n-1} \frac{(-1)^{m+1}}{m} \sum_{i_{1}<i_{2}<\cdots<i_{m}} \prod_{j=i_{1}}^{i_{m}} P_{j} \sum_{j=i_{1}}^{i_{m}} R_{j} \\
& I I=\sum_{m=1}^{n-1} \frac{(-1)^{m+1}}{m+1} \sum_{i_{1}<i_{2}<\cdots<i_{m}} \prod_{j=i_{1}}^{i_{m}} P_{j} \sum_{j=i_{1}}^{i_{m}} R_{j}
\end{aligned}
$$

\subsection{One Step Smart Predictive Algorithm Formulation for One Resource}

One step Smart Predictive is an improved solution over the Greedy solution that proceeds by determining a preferred network (network with highest expected profit $P_{i} R_{i}$ ) and then computing whether reserving the resource would have a higher expected value outcome than assigning through Greedy. This procedure has three steps:

(1) Calculate Greedy expected value.

(2) Calculate $\max P_{i} R_{i}$, over the span of all networks.

(3) Adopt the strategy that maximizes the expected profit.

In other words, Smart Predictive Algorithm at every minute decides either to reserve the only resource for the network with highest predicted expected profit, $P_{i} R_{i}$, or to just leave it to Greedy. The following Theorem proves that the Smart Predictive algorithm is superior to Greedy. Simulation study demonstrated that Smart Predictive outperforms the Greedy algorithm.

THEOREM 3.1. Under the assumptions of known value and probability of break arrival per minute per network, independence between networks and $\max _{i=1,2, \cdots, n} P_{i} R_{i}=P_{1} R_{1}$ we have

$$
S P_{1}(S) \geq G_{1}(S)
$$

where

$$
S P_{1}(S)= \begin{cases}P_{1} R_{1} & P_{1}>\frac{I}{I I+R_{1}-\Delta R_{1}} \\ G_{1}(S) & \text { o.w. }\end{cases}
$$

PROOF. Under the assumption $P_{1}>\frac{I}{I I+R_{1}-\Delta R_{1}}$ we get $P_{1} I I+$ $P_{1} R_{1}-P_{1} \Delta R_{1}>I$ which results in $P_{1} R_{1}>P_{1} R_{1} \Delta+I-P_{1} I I$ and equivalently $P_{1} R_{1}>G_{1}(S)$. $\square$

\subsection{An Illustrative Example}

Herein, we give an example to better understand the behavior of the introduced Smart Predictive algorithm versus the Greedy algorithm. Assuming two networks $(n=2)$ and only one available resource $(k=1)$, the expected profit through the Greedy algorithm would be

$$
G_{1}(\{1,2\})=P_{1}\left(1-P_{2}\right) R_{1}+P_{2}\left(1-P_{1}\right) R_{2}+P_{1} P_{2}\left(\frac{1}{2} R_{1}+\frac{1}{2} R_{2}\right)
$$

Note that in the case of collision, the profit through the Greedy algorithm would be either $P_{1} P_{2} R_{1}$ or $P_{1} P_{2} R_{2}$ depending on which network goes to break first and consequently a suboptimal choice will be made in the case that network 2 goes to break first but 
$R_{1}>R_{2}$. However, the Smart Predictive algorithm would reserve the resource for network 1 and decline the request for resource of network 2 since $P_{1} R_{1}>P_{2} R_{2}$.

\subsection{Approximation and Quadratic Computation of Expected Greedy for for One Resource}

From equation (1), we can see that the expected Greedy for all possible subsets of breaking networks, just for a single resource, $k=1$, has $\sum_{m=1}^{|S|}\left(\begin{array}{c}|S| \\ m\end{array}\right)=2^{|S|}-1$ terms and the direct calculation has exponential computational complexity. In addition, direct calculation of the reformulated version of expected Greedy is computationally expensive as well. A way out this would be either approximating [7] the expected Greedy which is a trade off between accuracy and the complexity of calculation, or finding a way to recursively formulate the expected Greedy in order to reduce its complexity.

3.5.1 Approximation of Expected Greedy for for One Resource. Due to the fact that

$$
\sum_{i_{1}<i_{2}<\cdots<i_{m}} a_{i_{1}} a_{i_{2}} \cdots a_{i_{m}} \leq \frac{1}{m !\left(\sum_{i} a_{i}\right)^{m}} \text { for } a_{i} \geq 0,
$$

we have following upper bounds for $\Delta, I$ and $I I$

$$
\begin{aligned}
& \Delta \leq 1+\sum_{m=1}^{k-1} \frac{(-1)^{m}\left(\sum_{i=2}^{k} P_{i}\right)^{m}}{(m+1) !}, \\
& I \leq \sum_{i=2}^{k} P_{i} R_{i} \sum_{m=1}^{k-1} \frac{(-1)^{m+1}\left(\sum_{i=2}^{k} P_{i}\right)^{m-1}}{m !} \\
& I I \leq \sum_{i=2}^{k} P_{i} R_{i} \sum_{m=1}^{k-1} \frac{(-1)^{m+1}\left(\sum_{i=2}^{k} P_{i}\right)^{m-1}}{(m+1)(m-1) !}
\end{aligned}
$$

3.5.2 Quadratic Computation of Expected Greedy for for One Resource. By using a recursive formulation, we can compute the expected value of Greedy in $O\left(n^{2}\right)$ computational complexity. The recursion is performed on the size of the set of networks currently under consideration. Suppose we had a calculated value for expected Greedy for a set of networks $S_{\alpha}$. Then, if we could use that in a linear-time calculation for the value of expected Greedy for the set $S_{\beta}=S_{\alpha} \cup s$ for any network $s$ not already in $S_{\alpha}$, we could recurse through the set of networks not yet included and eventually calculate expected Greedy for the entire set of networks. For simplicity, we describe the algorithm with the assumption that the networks are numbered $\{1,2, \cdots, n\}$ and recurse by introducing the next numbered network at each step.

Let $G_{1}(n-1)$ be the value for expected Greedy if we include only the first $n-1$ networks $(\{1,2, \cdots, n-1\})$. If we introduce the network $n$, then either that network goes to break or it does not. If it doesn't, which has probability $1-P_{n}$, then the value for expected Greedy in this case is the same as the previous value, which we already know is $G_{1}(n-1)$. So, we only need to calculate the case for when the new network goes to break, which happens with probability $P_{n}$. Clearly, $G_{1}(1)=P_{1} R_{1}$, so we have a base case for our recursion.
Furthermore, the expected Greedy at step $n$ depends on the number of networks colliding at step $n-1$. To that end, let $C_{n}$ be the size of the set of networks in collision amongst the first $n$ networks, and define $E_{n}^{j}$ to be the amount of rewards when $j$ networks go to break $\left(C_{n}=j\right)$ among the first $n$ networks. In addition, we define $B_{n}^{j}=P\left(C_{n}=j\right), 0 \leq j \leq n, n \geq 1$, where $B_{n}^{j}$ is the Poisson Binomial distribution over our break arrival probabilities $P_{1}, P_{2}, \cdots, P_{n}$ and which has its own quadratic time recursive computation.

Therefore,

$$
\begin{aligned}
E_{n}^{j} & =P\left(C_{n-1}=j \mid C_{n}=j\right) E_{n-1}^{j} \\
& +P\left(C_{n-1}=j-1 \mid C_{n}=j\right) \frac{(j-1) E_{n-1}^{j-1}+R_{n}}{j} \\
& =\frac{\left(1-P_{n}\right) B_{n-1}^{j}}{B_{n}^{j}} E_{n-1}^{j}+\frac{P_{n} B_{n-1}^{j-1}}{B_{n}^{j}} \frac{(j-1) E_{n-1}^{j-1}+R_{n}}{j}
\end{aligned}
$$

By considering $G_{n}^{j}=B_{n}^{j} E_{n}^{j}$ (with $G_{n}^{0}=0$ ), we have

$$
G_{n}^{j}=\left(1-P_{n}\right) G_{n-1}^{j}+P_{n} \frac{(j-1) G_{n-1}^{j-1}+B_{n-1}^{j-1} R_{n}}{j}, \quad j>0
$$

and finally

$$
G_{1}(n)=\sum_{j=0}^{n} G_{n}^{j}
$$

The recursive calculation of the Poisson Binomial $B_{n}^{j}$ is:

$$
B_{n}^{j}=\left(1-P_{n}\right) B_{n-1}^{j}+P_{n} B_{n-1}^{j-1} .
$$

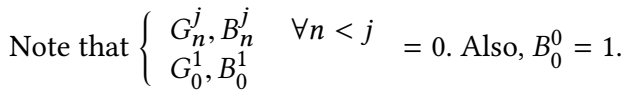

\subsection{Formulation of the Greedy Algorithm for an Arbitrary Number of Resources}

For any subset, $S$, of networks, we define $G_{k}(S)$ to be the expected reward of the Greedy algorithm serving $k$ resources at time $t$.

$$
\begin{aligned}
G_{k}(S) & =\sum_{I \in S_{\ell}, \ell \leq k} L_{S}(I) \sum_{j \in I} R_{j} \\
& +\sum_{I \in S_{k+1}} L_{S}(I) \frac{1}{\left(\begin{array}{c}
k+1 \\
k
\end{array}\right)} \sum_{J \in I_{k}} \sum_{j \in J} R_{j} \\
& +\sum_{I \in S_{k+2}} L_{S}(I) \frac{1}{\left(\begin{array}{c}
k+2 \\
k
\end{array}\right)} \sum_{J \in I_{k}} \sum_{j \in J} R_{j} \\
& +\cdots+\sum_{I \in S_{|S|}} L_{S}(I) \frac{1}{\left(\begin{array}{c}
|S| \\
k
\end{array}\right)} \sum_{J \in I_{k}} \sum_{j \in J} R_{j}
\end{aligned}
$$

It is obvious that direct calculation of the Greedy expected value has computational complexity that is exponential in the number of networks.

3.6.1 Quadratic Computation of Expected Greedy for for an Arbitrary Number of Resources. Here we generalize the recursive formula for calculation of expected Greedy for any subset of networks and an arbitrary number of resources $k$. Considering, $B_{n}^{j}$ : probability of $j$ networks go to break out of $n, E_{n}^{j}$ : profit when $j$ networks 
go to break out of $n$, and $G_{n}^{j}$ : expected profit when $j$ networks break out of $n$, we have

$$
G_{n}^{j}= \begin{cases}\left(1-P_{n}\right) G_{n-1}^{j}+P_{n}\left(G_{n-1}^{j-1}+B_{n-1}^{j-1} R_{n}\right) & j \leq k \\ \left(1-P_{n}\right) G_{n-1}^{j}+P_{n} \frac{(j-1) G_{n-1}^{j-1}+k B_{n-1}^{j-1} R_{n}}{j} & j>k\end{cases}
$$

and finally

$$
G_{k}(n)=\sum_{j=0}^{n} B_{n}^{j} E_{n}^{j}=\sum_{j=0}^{n} G_{n}^{j}
$$

\subsection{One step and Multi-step Smart Predictive Algorithm Formulation for an Arbitrary Number of Resource}

The Smart Predictive algorithm can be generalized to accommodate more than one resource available at a time for any subset, $S=$ $\{1,2, \cdots, n\}$, of networks and is called "one step Smart Predictive algorithm". For instance, in case of two resources at each minute, $k=2$, and under the condition $P_{1} R_{1}>P_{2} R_{2}>\cdots>P_{n} R_{n}$, here are the possible decisions:

(1) Do Greedy on both resources, so the expected reward would be $G_{2}(S)$.

(2) Reserve one resource and do the Greedy on the second one, which will result in expected reward of

$$
\max _{i=1,2, \cdots, n}\left(P_{i} R_{i}+G_{1}(S \backslash i)\right) .
$$

(3) Reserve both resources and get $P_{1} R_{1}+P_{2} R_{2}$ as expected reward.

Finally, the chosen Strategy will be the one maximizing overall expected profit at that minute:

$$
\max \left(G_{2}(S), \max _{i=1,2, \cdots, n}\left(P_{i} R_{i}+G_{1}(S \backslash i)\right), P_{1} R_{1}+P_{2} R_{2}\right)
$$

Simulation in the next section demonstrated that this algorithm is superior to the Greedy algorithm. Furthermore, the efficiency of this algorithm can be improved by including a mixed strategy called "multi-step Smart Predictive algorithm".

The idea behind this mixed strategy is very simple. For example in the case $k=2$, similar to the one step Smart Predictive algorithm, it still has three possible choices:

(1) Do Greedy on both resources.

(2) Reserve one resource and do the Greedy on the second one.

(3) Reserve both resources.

However, the way we reserve resource/s will be updated. Following are the expected profits based on each potential decision:

(1) $\mathrm{EP} 1=G_{2}(S)$

(2) $\mathrm{EP} 2=\left\{\begin{array}{l}\max _{i=1,2, \cdots, n}\left(P_{i} R_{i}+G_{1}(S \backslash i)\right) \\ \text { or } \max _{i, j=1,2, \cdots, n, i<j}\left(G_{1}(\{i, j\})+G_{1}(S \backslash\{i, j\})\right)\end{array}\right.$

(3) $\mathrm{EP} 3=\left\{\begin{array}{l}P_{1} R_{1}+P_{2} R_{2} \\ \text { or } \max _{i, j, m, l=1,2, \cdots, n, i<j<m<l}\left(G_{2}(\{i, j, m, l\})\right)\end{array}\right.$

Where $G_{1}(\{i, j\})=P_{i}\left(1-P_{j}\right) R_{i}+P_{j}\left(1-P_{i}\right) R_{j}+\frac{P_{i} P_{j}}{2}\left(R_{i}+R_{j}\right)$.
Finally, the chosen Strategy will be the one maximizing overall expected profit at that minute:

$\max (E P 1, E P 2, E P 3)$

Notice that in part (2), instead of reserving just for the network with highest $P_{i} R_{i}+G_{1}(S \backslash i)$, we have the option to reserve for the two networks with highest $G_{1}(\{i, j\})+G_{1}(S \backslash\{i, j\})$ and then do Greedy between them. In the same way, in part (3) we can either reserve both resources for the two networks with highest expected profit or reserve for the first four highest networks and then do Greedy between them.

In the case $k=1$, multi-step allows for reserving for the two networks with highest expected profits and doing Greedy between them as well as reserving the single resource for the first network with highest expected profit. Generalization to more than two resources is also derived, but it is not presented here for notational simplicity and the fact that in practice it is not that likely that there will be more than a couple of resources available at a time, since each resource means reserving three or four ad delivery channels and bandwidth constraints will make these scarce.

\section{METHOD EVALUATION}

In this section, we evaluate our methods on historical data. To provide a fair assessment, we have to apply the proposed algorithm in a way that it does not know about the future but only the past, thereby simulating the real setting in which the algorithm runs. The optimal algorithm is formulated for the case of known probabilities of break arrival per unit of time per network, however, we do not know these probabilities and must estimate them from historical data. Due to the time dependency of break arrivals, probabilities are estimated dynamically. The data set is divided into training and testing sets to ensure realistic simulations. The training set is used to estimate the probability distribution of next break arrivals, from which we estimate the probability of networks going to break within the next minute given there was no break to this time (the hazard function). The testing set is used for evaluation of the methodology. Estimated probabilities from the data and the known reward associated with each network per minute help us to simulate the decision procedure within Smart Predictive.

It is important to notice that we are not interested in point prediction of when the next break happens, but instead the whole distribution of next break arrival. Hence, we are concerned with probability forecasts, so our predictand is a probability, the probability of break arrival in the next minute. There are various methods for probability distribution estimation, but what is considered here is obtaining probabilities based on a sequence of conditional density functions for any given interval between breaks, by combining kernel and nearest-neighbor methodology with borrowing some strength in the empirical Bayes flavor. The details of prediction techniques are beyond the scope of this paper and are to appear elsewhere.

\subsection{The Data Setting}

Here, the historical break data from the San Francisco area over eighty-three days (Aug. 1- Oct. 22, 2007) is combined with viewership data from San Francisco for the experiment. The data is divided 
into the first 61 days, which is used for estimation of the probability distribution of the intervals between breaks, and last 22 days that are used for evaluation of the methodology.

The break data contains the ad breaks times and break durations, to the second, for each network on each day, permitting calculation of waiting times between breaks. Viewership data is represented as a relative estimated count of viewing by households of television stations and cable programming available in the region by fifteen minute period. Viewership is assumed fixed within each fifteen minutes. So, the value of each break might be evaluated through combining break data and the viewership data. In total, the break data and viewership data of 27 networks are matched.

\subsection{Results}

The performance of the Greedy algorithm verses the Smart Predictive algorithm (one step and multi-step) based on approximation method and quadratic recursive method with one and two resources are compared.

Table 1: Smart Predictive Algorithm vs Greedy Algorithm Performance for $k=1$

\begin{tabular}{lclcc}
\hline & Expected Profit & Improvment & Max & Collision \\
\hline \hline & & & & \\
Greedy & $65,234,469$ & & 7,822 \\
SP-Approximation & $66,671,599$ & $2.203 \%$ & & \\
SP-One step & $66,746,608$ & $2.31801 \%$ & & \\
SP-Multi-step & $70,419,812$ & $\mathbf{7 . 9 4 8 7 8 \%}$ & & \\
\hline \hline
\end{tabular}

From Table 1, the summation of delivered impressions over the 22 days of testing for the Greedy algorithm is 65,234, 469 million. Using approximation techniques, the amount of delivered impressions through the Smart Predictive algorithm is 66, 671, 599 million, which is very close to the $66,746,608$ million calculated based on exact method (quadratic recursive). This shows that the approximation bound is very tight and accurate. In addition, it has the advantage of being fast in terms of computation. Multi-step Smart Predictive algorithm delivered 70,419,812 million impressions. These results are consistent with the theory, $S P_{1}(S) \geq G_{1}(S)$. In terms of improvement, Smart Predictive using the multi-step methodology exhibits the most improvement over the Greedy algorithm (7.94878\%) which results in 5,185,343 million more impression deliveries.

Over the whole considered minutes in 22 days, 7, 822 times we had cases that more than one network asked for resource in an overlapping minute and the maximum number of networks going into break around the same time was 9 networks.

Table 2: Smart Predictive Algorithm vs Greedy Algorithm Performance for $k=2$

\begin{tabular}{lcccc}
\hline & Expected Profit & Improvment & Max & Collision \\
\hline \hline & & & & \\
Greedy & $94,88,7104$ & & 9 & 3,035 \\
SP-One step & $95,544,386$ & $0.6926994 \%$ & & \\
SP-Multi-step & $96,393,755$ & $\mathbf{1 . 5 8 7 8 3 6 \%}$ & & \\
\hline \hline
\end{tabular}

Table 2 summarizes the result of the resource allocation problem considering all 27 networks and two resources available per minute. It is important to notice that here we are considering actually four resources, two for even minutes and two for odds minutes. Smart Predictive using the multi-step method exhibits the most improvement over the Greedy algorithm which results in 1, 506, 651 million more impressions delivered which is equivalent to a $1.5 \%$ improvement. Note that in case of $k>1$ we cannot do much of an improvement over the Greedy, since for most of the minutes we have enough resource to support the incoming break. The Smart Predictive is the most useful when we have collisions. In tests with more networks competing in the system, it is expected that Smart Predictive provides substantial benefits in $k>1$ cases.

Over the whole considered minutes in 22 days, 3, 035 times we had cases that more than two network asked for resource in an overlapping minute and the maximum number of networks going into break around the same time was 9 networks.

In addition, assuming that Greedy is an instant algorithm in terms of the execution time, following is the summary of execution time of Smart Predictive under different strategies over 27 networks and 22 days of testing data. The scripts are written in the R language, without optimization.

Table 3: Execution time of each step of Smart Predictive under different strategies (in seconds)

\begin{tabular}{lllll}
\hline & Min & Median & Mean & Max \\
\hline \hline SP-Approximation, $k=1$ & 0.00 & 0.00 & 0.0012 & 0.13 \\
SP-One step, $k=1$ & 0.00 & 0.02 & 0.0149 & 0.31 \\
SP-Multi-step, $k=1$ & 0.00 & 0.03 & 0.026 & 0.38 \\
SP-One step, $k=2$ & 0.01 & 0.05 & 0.0582 & 0.39 \\
SP-Multi-step, $k=2$ & 0.04 & 0.08 & 0.0828 & 0.63 \\
\hline \hline
\end{tabular}

The approximation method to evaluate the Smart Predictive algorithm is the fastest, followed by one step and multi-step for $k=1$. In the case of two resources available, $k=2$, it is obvious that the multi-step takes more execution time but the gained profit over the one step is more significant. In addition, the amount of processing time required in all cases is well within the computational capabilities of live systems.

\section{CONCLUSION}

A new probabilistic algorithm for bandwidth and resource allocation for addressable advertising in traditional TV with application in Mobile TV platform is introduced. Its $O\left(n^{2}\right)$ implementation together with an approximate and exact recursive solutions are developed. The performance of the proposed methods were assessed on real historical data from the traditional TV industry, which resulted in significant profitability in terms of advertising over the current method in use in the industry. In fact, the multi-step Smart Predictive algorithm improved revenue by $7.94878 \%$ in case of single resource $(k=1)$, and increased the profitability by $1.5 \%$ in the case of $k=2$. In addition, the processing times for all proposed methods are assessed and are well within the computational capabilities of live systems. We emphasize that transmitting addressable advertising over a mobile TV platform via LTE broadcast 
has relevant structural similarities to delivering this advertising over traditional television systems, and so these results from the one area are applicable to the other.

\section{ACKNOWLEDGMENTS}

The authors would like to thank David Ballantyne for his assistance in formulating the quadratic formula of the Greedy algorithm.

This work is supported by Mitacs and INVIDI Technologies Corporation.

\section{REFERENCES}

[1] R. Adany, S. Kraus, and F. Ordonez. 2010. Uncertain personal advertisement allocation for Mobile TV. In Proceedings of the 8th International Conference on Advances in Mobile Computing and Multimedias.

[2] R. Adany, S. Kraus, and F. Ordonez. 2013. Allocation algorithms for personal TV advertisements. Multimedia Systems 19 (2013), 79-93.

[3] B. J. Anderson, D. C. Wilson, and D. A. Boulet. 2006. Targeted impression model for broadcast network asset delivery. Google Patents (CA Patent App. CA 2,594,003) (2006).

[4] V. Dureau. 2004. Addressable advertising on digital television. In Proceedings of the 2nd European conference on interactive television: enhancing the experience. Brighton UK.

[5] W. Feller. 1911. An introduction to probability theory and its application (3rd. ed.) Wiley, New York, NY.

[6] B. Kitts and A. Dyng. 2014. A comparison of algorithms for TV ad targeting. IEEE International Conference on Data Mining (2014).

[7] M. A. Kouritzin and S. Sadeghi. 2015. Convergence Rates and Decoupling in Linear Stochastic Approximation Algorithms. SIAM fournal on Control and Optimization 53-3 (2015), 1484-1508.

[8] S. Ross. 2014. Introduction to Probability Models (11th ed.). Academic Press. 